Research Paper

\title{
Upregulation of miR-335-3p by NF-KB Transcriptional Regulation Contributes to the Induction of Pulmonary Arterial Hypertension via APJ during Hypoxia
}

\author{
Junming Fan*, Xiaofang Fan*, Hui Guang*, Xiaoqiong Shan, Qiuyun Tian, Fukun Zhang, Ran Chen, \\ Fangzhou Ye, Hui Quan, Haizeng Zhang, Lu Ding, Zhuohui Gan, Feng Xue, Yongyu Wang, Sunzhong \\ Mao, Lianggang $\mathrm{Hu}$, and Yongsheng Gong ${ }^{\bowtie}$ \\ Institute of Hypoxia Medicine, School of Basic Medical Sciences, Wenzhou Medical University, Wenzhou, Zhejiang, 325035, China. \\ *These authors contributed equally to this work. \\ $\square$ Corresponding author: Yongsheng Gong, Institute of Hypoxia Medicine, School of Basic Medical Sciences, Wenzhou Medical University, Wenzhou, Zhejiang, \\ 325035, China. E-mail: fxbwzmc@126.com (YS Gong); Tel: (+86)577 86699521
}

(C) The author(s). This is an open access article distributed under the terms of the Creative Commons Attribution License (https://creativecommons.org/licenses/by/4.0/). See http://ivyspring.com/terms for full terms and conditions.

Received: 2019.03.01; Accepted: 2019.10.15; Published: 2020.01.01

\begin{abstract}
Pulmonary arterial hypertension (PAH) is a cardiopulmonary disease that can lead to heart failure and eventually death. MicroRNAs (miRs) play essential roles during PAH progression; however, their exact mechanism of action remains unclear. Apelin is a small bioactive peptide with a key protective function in the pathogenesis of PAH mediated by binding to the APJ gene. The aim of the present study was to investigate the role of miR-335-3p in chronic normobaric hypoxia $(\mathrm{CNH})$-induced $\mathrm{PAH}$ in mice and the potential underlying regulatory mechanism. Adult male C57BL/6 mice were exposed to normoxia $\left(\sim 21 \% \mathrm{O}_{2}\right)$ or $\mathrm{CNH}\left(\sim 10 \% \mathrm{O}_{2}, 23 \mathrm{~h} / \mathrm{d}\right)$ for 5 weeks. MiR-335-3p was significantly increased in lung tissue of $\mathrm{CNH}$-induced $\mathrm{PAH}$ mice. Blocking miR-335-3p attenuated $\mathrm{CNH}$-induced $\mathrm{PAH}$ and alleviated pulmonary vascular remodeling. Bioinformatics analysis and luciferase reporter assay indicated that nuclear factor-kappa beta (NF-KB) acted as a transcriptional regulator upstream of miR-335-3p. Pyrrolidine dithiocarbamate treatment reversed the $\mathrm{CNH}$-induced increase in miR-335-3p expression and diminished $\mathrm{CNH}$-induced $\mathrm{PAH}$. Moreover, $\mathrm{p} 50^{-1-}$ mice were resistant to $\mathrm{CNH}$-induced PAH. Finally, APJ was identified as a direct targeting gene downstream of miR-335-3p, and pharmacological activation of $\mathrm{APJ}$ by its ligand apelin-13 reduced $\mathrm{CNH}$-induced $\mathrm{PAH}$ and improved pulmonary vascular remodeling. Our results indicate that NF-KB-mediated transcriptional upregulation of miR-335-3p contributes to the inhibition of APJ and induction of PAH during hypoxia; hence, miR-335-3p could be a potential therapeutic target for hypoxic PAH.
\end{abstract}

Key words: NF-kB; Pulmonary arterial hypertension; miR-335-3p; APJ; Hypoxia

\section{Introduction}

Pulmonary arterial hypertension (PAH) is a devastating lung disease characterized by increased pulmonary vascular resistance, which can lead to right heart failure and even death $[1,2]$. Structural and functional changes in pulmonary arterial smooth muscle cells (PASMCs) responsible for pulmonary vascular remodeling are thought to represent the main cellular mechanism underlying the development of PAH [3-5]. However, the pathogenesis of PAH has yet to be fully delineated.

MicroRNAs (miRs), 20-26 nucleotide-long single-stranded non-coding RNA molecules, participate mainly in the inhibition of post-transcriptional gene expression by interacting with the 3'-untranslated regions (3'-UTR) of their downstream target mRNAs [6, 7]. A growing number 
of studies have shown that miRs are involved in diverse key biological processes including cancer, inflammatory responses, and cellular function [8,9]. Recently, miRs have been strongly associated with development and progression of $\mathrm{PAH}$ and the modulation of its pathophysiological function $[10,11]$. Moreover, they exhibit essential roles in both proliferation and migration of PASMCs, as well as vascular remodeling in $\mathrm{PAH}$ during hypoxia, as have been reported by a number of elegant studies [12-22]. Although miRs are known major upstream regulators of many key target genes involved in hypoxic PAH development, the transcriptional regulation of miRs by critical effectors on PAH during hypoxia is less clear. Therefore, it is important to uncover how miR activation is associated with other well-defined transcriptional factors in the progression of hypoxic PAH.

The expression of miRs in response to environmental stimuli is often subjected to regulation by transcriptional factors including nuclear factor-kappa beta (NF-kB) [23-26]. NF-kB plays an essential role in the modulation of PAH [27-32], and NF- $\mathrm{KB}$ activation is one of the primary hypoxia-driven signals to promote pulmonary arterial obliteration, inflammation, and reduced immune regulation in the context of severe obliterative PAH [33]. Although miRs are becoming increasingly recognized as important regulators of $\mathrm{PAH}$, comparatively little is known about the specific role of the NF-kB pathway in $\mathrm{miR}$ regulation during $\mathrm{PAH}$ pathogenesis. These facts led us to hypothesize that NF-kB-mediated regulation of miRs might contribute to the modulation of PAH during hypoxia.

In this study, we first screened and addressed the potential role of miR-335-3p in $\mathrm{CNH}$-induced $\mathrm{PAH}$ in mice. We then treated mice with an NF-kB inhibitor, pyrrolidine dithiocarbamate (PDTC), and utilized NF-kB knockout ( $\mathrm{p} 50^{-/-}$) mice to test whether the essential role of NF-KB in $\mathrm{CNH}$-induced PAH depended on upregulation of miR-335-3p. Finally, we investigated whether NF-kB-mediated transcriptional upregulation of miR-335-3p contributed to the inhibition of APJ, a downstream target of miR-335-3p, during the development of $\mathrm{CNH}$-induced $\mathrm{PAH}$.

\section{Materials and methods}

\section{Animals}

Adult male C57BL/6 mice aged 10-12 weeks were purchased from the Animal Center of Wenzhou Medical University (Wenzhou, Zhejiang, China). The animals were housed in groups of four in standard cages $(30 \times 20 \times 12 \mathrm{~cm})$, maintained at ambient temperature $\left(22 \pm 1^{\circ} \mathrm{C}\right)$ and humidity $(55 \pm 5 \%)$, and were kept on a 12-hour light/dark cycle (lights on at 07:00-19:00 hours) with ad libitum access to mouse chow and water. The animals were allowed to acclimatize in the animal facility for 1 week before experimental manipulation. All efforts were made to minimize animal suffering.

\section{Chronic normobaric hypoxia (CNH) exposure}

Mice were randomly divided into Normoxia control and $\mathrm{CNH}$ groups (N=5-8 per group). For $\mathrm{CNH}$ exposure, mice were placed carefully in a normobaric hypoxic chamber with a fraction of inspired oxygen $\left(\mathrm{FIO}_{2}\right)$ of $\sim 0.1,23 \mathrm{~h}$ per day, for five weeks. Mice in Normoxia group were kept in a normobaric chamber at sea level with $\mathrm{FIO}_{2}$ of $\sim 0.21$, as we described previously [34]. Cages were cleaned once daily between 10:00 and 11:00 h.

\section{MiR-335-3p antagomir treatment in CNH-induced PAH in mice model}

To investigate whether there is a preventive effect of miR-335-3p on $\mathrm{CNH}$-induced $\mathrm{PAH}$, mice were randomly divided into four groups $(\mathrm{N}=5-8$ each group): 1) Normoxia+miR-335-3p scramble control, 2) Normoxia + miR-335-3p antagomir, 3) $\mathrm{CNH}+\mathrm{miR}-335-3 \mathrm{p}$ scramble control, 4) $\mathrm{CNH}+$ miR-335-3p antagomir. MiR-335-3p antagomir or miR-335-3p scramble control were injected intravenously (tail vein, $5 \mathrm{nmol}$ at $0.1 \mathrm{ml}$ ) at day 0,7 , 14,21 , and 28, and the mice were sacrificed at day 35 .

To test whether there is a therapeutic effect of miR-335-3p on CNH-established PAH model, mice were exposed to $\mathrm{CNH}$ for 5 weeks to induce $\mathrm{PAH}$, followed by housing at normoxia condition for remaining 10 weeks. Therapeutic experiment with miR-335-3p antagomir administration was undertaken at 11,12,13, and 14 weeks, and the animals were sacrificed at 15 weeks. MiR-335-3p antagomir was synthesized by Ribobio Co., Ltd. (Guangzhou, China).

\section{Pyrrolidine dithiocarbamate (PDTC) treatment}

Mice were randomly divided into four groups (N=5-8 per group): 1) Normoxia ${ }^{+}$vehicle; 2) Normoxia+PDTC; 3) $\mathrm{CNH}+$ vehicle; and 4) $\mathrm{CNH}+\mathrm{PDTC}$. Mice in the Normoxia+PDTC and $\mathrm{CNH}+\mathrm{PDTC}$ groups were subcutaneously injection of PDTC (50 mg.kg-1 day $^{-1}$ ), twice daily (10:00 and 18:00 h), and those in the Normoxia+vehicle and $\mathrm{CNH}+$ vehicle groups were subcutaneously injected with the same volume of vehicle as PDTC treatment, and exposed to normoxia or $\mathrm{CNH}$ treatment, as described above. PDTC was freshly dissolved in normal saline each day before injection. 


\section{Apelin-13 treatment}

Mice were randomly divided into four groups (N=5-8 per group): 1) Normoxia+vehicle, 2) Normoxia+apelin-13, 3) $\mathrm{CNH}+$ vehicle, 4) $\mathrm{CNH}+$ apelin-13. Mice in Normoxia+apelin-13 and $\mathrm{CNH}+$ apelin-13 groups were intraperitoneal injection with apelin-13 (15 ng/mice/day), and mice in Normoxia+vehicle and $\mathrm{CNH}^{+}$vehicle groups were intraperitoneal injection with the same volume of vehicle as apelin-13 treatment, and exposed to normoxia or $\mathrm{CNH}$ treatment, as described above. Apelin-13 was freshly prepared in normal saline $(\mathrm{pH}$ 7.4) each day before injection (10:00 h).

\section{Measurements of RVSP}

The degree of $\mathrm{PAH}$ was recorded by measuring right ventricular systolic pressure (RVSP) with right heart catheterization as we previously described [12]. In brief, the animals were anesthetized by intraperitoneal injection with pentobarbital (30 $\mathrm{mg} / \mathrm{kg}$ ), ventilated through a transtracheal catheter, and laid in a supine position on a heating platform. A microcatheter was inserted gently through jugular vein into right ventricle (RV) and then pulmonary artery. After an equilibration period for 30 minutes, RVSP was recorded on a physiological recorder (PowerLab) via a transducer (PowerLab 8 passages electrophysiolograph; ADI) connected to the micro-catheter.

\section{Assessment of right ventricular hypertrophy (RVH)}

After RVSP measurement, the animals were sacrificed and hearts were collected. Atrium was trimmed and the free wall of RV was separated from the left ventricle and septum $(\mathrm{LV}+\mathrm{S})$. RV and $\mathrm{LV}+\mathrm{S}$ of each heart were weighted, and the ratio of $\mathrm{RV} /(\mathrm{LV}+\mathrm{S})$ was calculated to assess RVH. The animals were sacrificed and the lung tissues were harvested and stored at $-80^{\circ} \mathrm{C}$ until further analysis.

\section{Morphometric analyses of pulmonary vascular remodeling}

To evaluate pulmonary arterial muscularization, lungs of mice were infused and fixed with $4 \%$ paraformaldehyde and embedded in paraffin, and lung sections $(5 \mu \mathrm{m})$ were prepared and stained with Masson trichrome stain as we previously described [35]. Distal small arteries with an external diameter between $50 \mu \mathrm{m}$ and $100 \mu \mathrm{m}$ for each section were captured using Nikon eclipse biological microscopy (Nikon, Japan) under high magnification. Arterial diameter and area were assessed using Nikon NIS-Elements basic research software for quantitative analyses, and vessel wall area/total cross area was calculated. Morphometric analysis was performed by the observer who was blinded to the experimental treatments.

\section{Microarray processing and analysis}

Microarray processing and analysis for miRs was performed by the Ribobio Co., Ltd. (Guangzhou, China). Briefly, total RNA was extracted using TRIzol reagent (Invitrogen). RNA quality and quantity were measured using a NanoDrop spectrophotometer (ND-1000, NanoDrop Technologies), and RNA integrity was determined using gel electrophoresis. RNA was then reverse-transcribed using the TruSeq RNA Library Prep Kit (Illumina, CA, USA). The libraries were subjected to quality validation using the Agilent 2200 TapeStation, and then paired-end sequenced using HiSeq 2500 (Illumina). The resulting reads were mapped to the rn5 database using TopHat2 [36]. Genes with an expression change $>1.5$-fold were clustered and shown in a heat map (log2 scale) using NetWalker62.

\section{Quantitative real-time polymerase chain reaction (qRT-PCR)}

Total RNA was isolated from mice lung tissues using TRIzol reagent (Invitrogen) and was reversely transcribed into single stranded complementary DNA with PrimeScript RT reagent Kit (Qiagen) following the manufacturer's instructions. Amplification and quantification were carried out with SYBR Premix Ex Taq II (Qiagen), and were processed using Applied Biosystems 7500 Real-time PCR System. The primers for pri-miR-335-3p and miR-335-3p and endogenous control RNU6B (U6) were synthesized by Ribobio Co., Ltd. (Guangzhou, China). All samples were amplified in triplicate. The relative mRNA expression levels were quantified using the comparative cycle threshold (CT) method $\left(2^{-\Delta \Delta C T}\right)$ and expressed as the fold-change compared with a control.

\section{Western blotting}

Lung tissues were lysed in RIPA buffer (1\% Triton X-100, 0.5\% sodium deoxycholate, $0.2 \%$ SDS, $150 \mathrm{mM} \mathrm{NaCl}, 10 \mathrm{mM}$ Hepes, pH 7.3, 2 mM EDTA, and protease inhibitor mixture; Pierce), as we previously described [12]. After blocking, membranes were probed overnight at $4^{\circ} \mathrm{C}$ with the primary antibodies as follows: APJ (SC-33823; Santa Crutz, USA), Bax (14796; CST, USA), Caspase 3 (9662; CST, USA), Cleaved caspase 3 (9661; CST, USA), a-SMA (14968; CST, USA), CD31 (3528; CST, USA), and GAPDH (2118; CST, USA). HRP-conjugated anti-rabbit or anti-mouse (CST) was used to detect the primary antibodies. After washing, the protein bands were visualized using an enhanced 
chemiluminescence (Bio-Rad). The relative expression of the proteins was quantified using densitometric scanning and analyzed by the Imaging J System and expressed as percent of controls. Molecular mass was determined relative to protein markers (BioRad).

\section{Promoter and 3'-UTR luciferase reporter assays}

To investigate the transcriptional regulation of NF-kB on miR-335-3p, we constructed a wild-type luciferase reporter vector containing $2260 \mathrm{bp}$ of the mouse pre-miR-335-3p 5' proximal genomic region and a mutant luciferase reporter vector in which the NF-kB binding sites were deleted. In order to validate the binding of candidate miRs to the APJ mRNA, TargetScan algorithm (http://www.targetscan.org) was applied to predict downstream targets of miR-335-3p and the corresponding binding sites. APJ 3'-UTR was PCR amplified from mice genomic DNA with primer 5'-GCG GCT CGA GCG ATG AAG GAC TAG GGT GAA C-3' (forward) and 5'- AAT GCG GCC GCC AGA GCC CTC CAA GAA CAA A-3' (reverse), and inserted into pmirGLO dual-luciferase vector (Promega). To construct mutated 3'-UTR report vector, residues in the region that base-pairs with miR-335-3p seeding sequences were mutated by site-directed mutagenesis. The primers used were listed as follows: APJ-mut, 5'-ACA GGA TGT ACT TTT AAG GGT GAG CTT TTG TGA-3' (forward) and 5'-TTT TTC ATA AAA GTA GGC AAG AAA GTG GCC TC-3' (reverse). For luciferase assays, $5 \times 10^{6}$ HEK293T cells were co-transfected with either APJ reporter plasmids together with miR-335-3p agomir or scramble control, or p65 cDNA and the luciferase reporter constructs described above. Transfection was performed using Lipofectamine 2000 (Invitrogen, ThermoFisher Scientific) according to the manufacturer's instructions. Twenty-four hours after transfection, cells were harvested and luciferase activity assay was measured using the Dual-Luciferase Assay System according to the manufacturer's instructions (Promega), and each well had three replicates. Firefly luciferase activity was normalized to renilla luciferase activity.

\section{Statistical analysis}

Values are presented as the mean \pm standard error of means (s.e.m). All data were analyzed with One-way or Two-way ANOVA followed by a Tukey's post hoc test using the GraphPad Prism software. Student's t-test was applied for comparisons between two groups. Pearson's correlation coefficients was used to analyze the correlation between miR-335-3p and RVSP, and miR-335-3p and RV/(LV+S). Results were considered to indicate statistical significance at $p<0.05$

\section{Results}

\section{CNH exposure induces PAH and upregulates miR-335-3p expression in mouse lung tissue}

To assess whether $\mathrm{CNH}$ exposure could induce $\mathrm{PAH}$ in mice, we measured right ventricular systolic pressure (RVSP) and right ventricular hypertrophy (RVH). Mice exposed to $\mathrm{CNH}$ had a $48.2 \%$ higher RVSP $(p<0.01)$ relative to normoxia control animals (Figure 1A) and a $28.3 \%$ higher right ventricle $(\mathrm{RV}) /$ left ventricle plus septum $(\mathrm{LV}+\mathrm{S})$ ratio $(p<0.01)$ (Figure 1B).

To determine the role of miRs in the pathogenesis of $\mathrm{CNH}$-induced $\mathrm{PAH}$, we performed a microarray analysis on RNAs isolated from the lungs of mice that were exposed to $\mathrm{CNH}$ or normoxia (controls). The overall profiles of the differently expressed miRs are presented as a heatmap (Figure 1C). As shown in Figure 1D, miR-335-3p expression was enhanced in the lungs of mice exposed to $\mathrm{CNH}$ compared with those of normoxia controls. The microarray data were confirmed by mature miR-specific quantitative PCR analysis, which revealed a significant increase in miR-335-3p expression in the lungs of mice exposed to $\mathrm{CNH}$ (Figure 1E). Pearson correlation analysis revealed that the expression of miR-335-3p in mouse lungs analyzed by quantitative real-time PCR (qRT-PCR) correlated positively with RVSP (Figure $1 \mathrm{~F}, r=0.92$, $p=0.002$ ) and $\mathrm{RV} /(\mathrm{LV}+\mathrm{S})$ (Figure 1G, $r=0.715$, $p=0.024)$. We also established a reproducible CNH-induced PAH rat model $\left(\mathrm{FiO}_{2} \sim 10 \%, 23 \mathrm{~h}\right.$ per day, for 2 weeks), as shown in Figure S1. Results of microarray and qRT-PCR analysis revealed that $\mathrm{CNH}$ exposure enhanced miR-335 expression in rat lungs (D-F in Figure S1), and that the latter correlated positively with mPAP ( $G$ in Figure S1, $r=0.861$, $p=0.033$ ) and $\mathrm{RV} /(\mathrm{LV}+\mathrm{S})(\mathrm{H}$ in Figure $\mathrm{S} 1, r=0.986$, $p=0.001)$. Together, these results indicate that $\mathrm{CNH}$ treatment induces PAH and right heart dysfunction as well as marked changes in miR-335-3p expression in mouse lungs.

\section{Blocking miR-335-3p abrogates the development of CNH-induced PAH in mice}

Given that miR-335-3p expression was increased in the lungs of $\mathrm{CNH}$-induced PAH mice (Figure 1D, Figure 1E), we tested whether blocking miR-335-3p could exert a protective effect during $\mathrm{CNH}$-induced $\mathrm{PAH}$ in mice. To this end, we administered $50 \mu \mathrm{l}(5 \mu \mathrm{M}$ concentration) of miR-335-3p antagomir (or scrambled control) at day-0, day-7, day-14, day-21, and day-28 after $\mathrm{CNH}$ exposure, and the mice were 
kept in $\mathrm{CNH}$ for total 5 weeks (Fig. 2A). Two-way ANOVA indicated that there was a significantly different effect on RVSP between $\mathrm{CNH}\left(\mathrm{F}_{(1,13)}=10.544\right.$; $p=0.009$ ) and $\mathrm{CNH}$ with antagomir treatment $\left(\mathrm{F}_{(1,13)}=4.457 ; p=0.049\right)$, while the antagomir alone had no significant effect $\left(\mathrm{F}_{(1,13)}=4.194 ; \quad p=0.068\right)$. A significant difference was observed also regarding the effect of $\mathrm{CNH}\left(\mathrm{F}_{(1,16)}=11.402 ; p=0.005\right)$, antagomir $\left(\mathrm{F}_{(1,16)}=9.728 ; \quad p=0.008\right)$, and $\mathrm{CNH} \times$ antagomir $\left(F_{(1,16)}=4.672 ; p=0.05\right)$ on the $\mathrm{RV} /(\mathrm{LV}+\mathrm{S})$ ratio. Post hoc analysis indicated that $\mathrm{CNH}$ significantly increased RVSP from $23.2 \pm 2.1$ to $32.7 \pm 1.8 \mathrm{mmHg}$ and $\mathrm{RVH}$ index from $0.22 \pm 0.02$ to $0.29 \pm 0.01$. In contrast, preventive administration of miR-335-3p antagomir significantly decreased RVSP from $32.7 \pm 1.8$ to $25.4 \pm 1.1$ $\mathrm{mmHg}$ and RVH index from $0.29 \pm 0.01$ to $0.24 \pm 0.02$ (all $p<0.05$ ) (Figure 2B, Figure 2C). The results of qRT-PCR showed that the endogenous miR-335-3p levels in lung tissues were significantly reduced when antagomir was administrated, as shown in Figure 2D.

Next, we evaluated the role of miR-335-3p in the regulation of $\mathrm{CNH}$-induced cellular proliferation and apoptosis in the lungs. We observed that $\mathrm{CNH}$ caused a modest increase in expression levels of proliferating cell nuclear antigen (PCNA) and the PASMCs marker alpha smooth muscle actin (a-SMA). It also caused a reduction in apoptosis manifested by a significant decrease in total caspase 3, cleaved caspase 3, and Bax expression levels. $\mathrm{CNH}$-induced PASMCs proliferation and apoptosis were reversed after the administration of miR-335-3p antagomir (Figure 2E, Figure 2F). This finding suggests that miR-335-3p stimulates lung PASMCs proliferation while inhibiting their apoptosis following $\mathrm{CNH}$ exposure in vivo. Histological analysis by hematoxylin and eosin and Masson trichrome staining of lung sections showed that, in comparison to the scrambled group, $\mathrm{CNH}$ significantly increased vascular remodeling, whereas miR-335-3p antagomir significantly prevented $\mathrm{CNH}$-induced pulmonary vascular remodeling in mice (Figure 2G, Figure 2H).

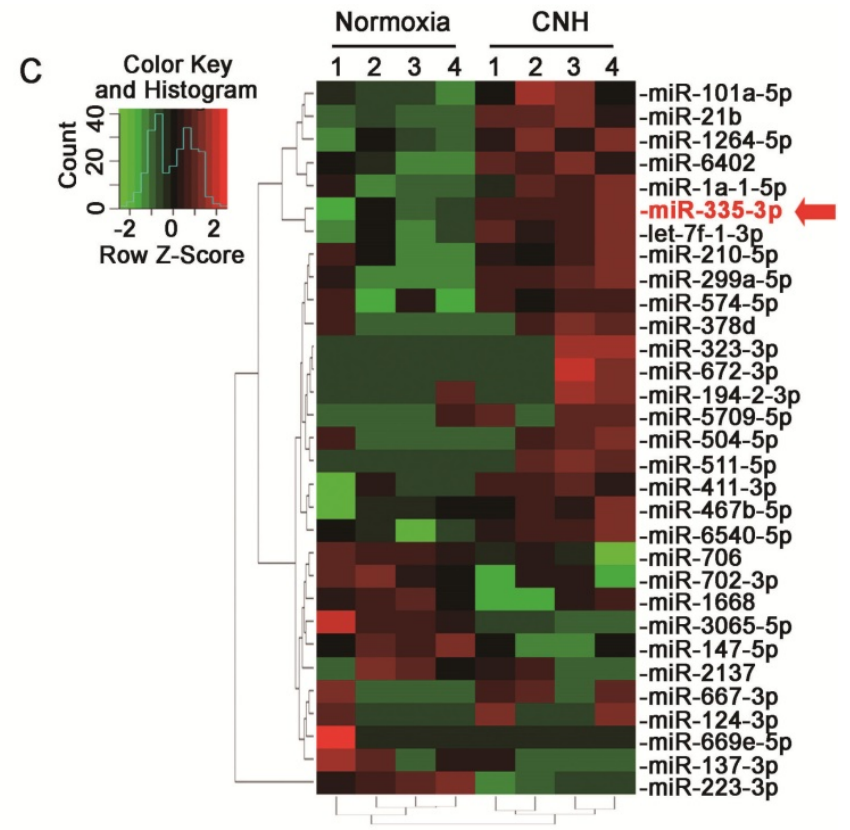

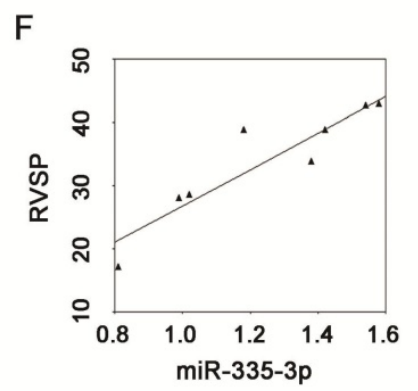
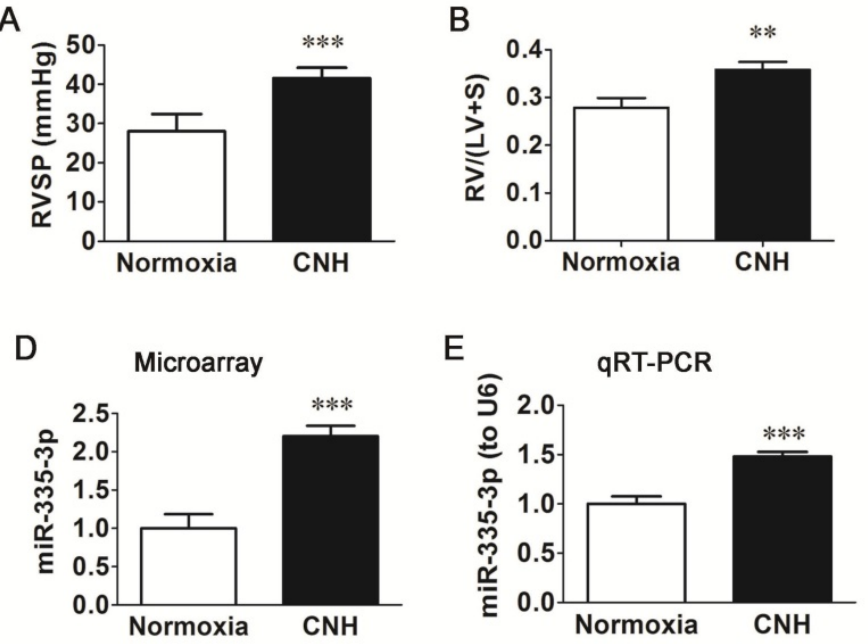

Figure 1. Chronic normobaric hypoxia (CNH) exposure induces pulmonary arterial hypertension (PAH) and upregulates miR-335-3p expression in the lungs of mice. CNH treatment significantly increased RVSP (A) and RV/(LV+S) (B) (N=5-8 per group). (C) Heatmap and sample clustering analysis of the differential expressed miRs in the lungs of mice ( $\mathrm{N}=4$ per group). Each row represents a miR and each column represents a sample. (D) Microarray and (E) quantitative RT-PCR analysis of miR-335-3p expression in the lungs of mice. (F) Correlation analysis between miR-335-3p and RVSP, and (G) Correlation analysis between miR-335-3p and $\mathrm{RV} /(\mathrm{LV}+\mathrm{S})(\mathrm{N}=4$ per group). Values shown are means \pm s.e.m. $* * p<0.01, * * * p<0.001$ vs. Normoxia. 
A

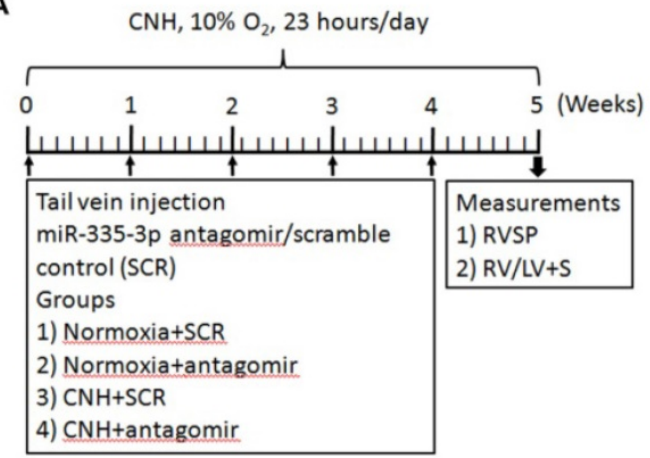

B

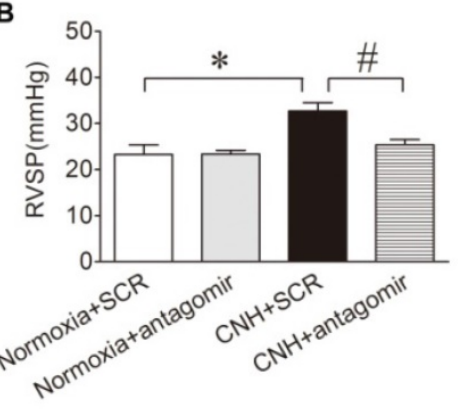

C

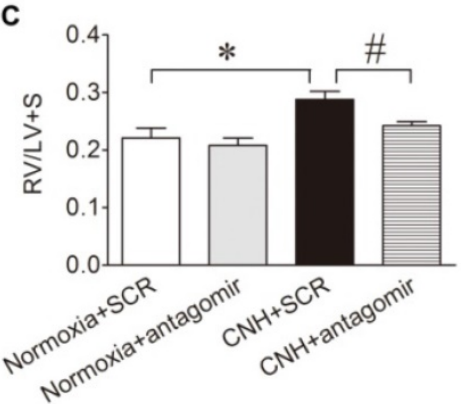

D

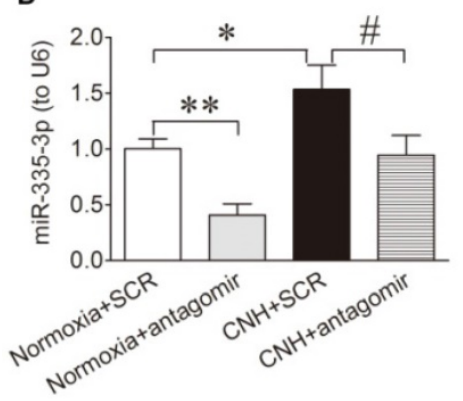

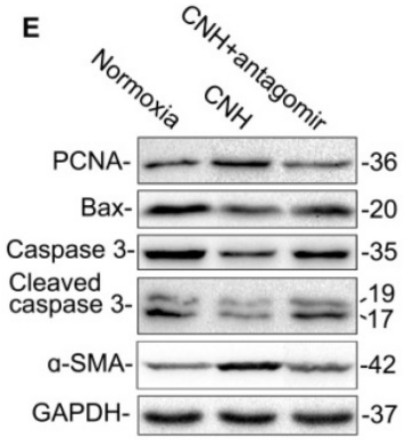

$\mathrm{CNH}+\mathrm{SCR}$

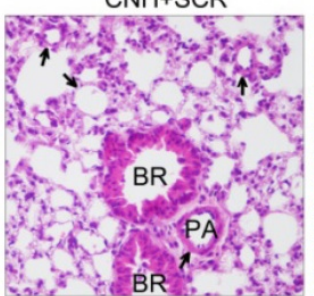

$\mathrm{CNH}+\mathrm{SCR}$

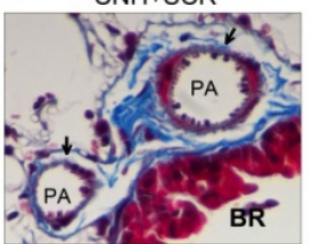

$\mathrm{CNH}+$ antagomir

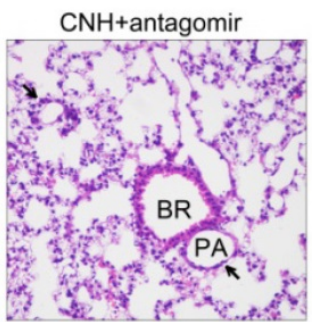

$\mathrm{CNH}+$ antagomir

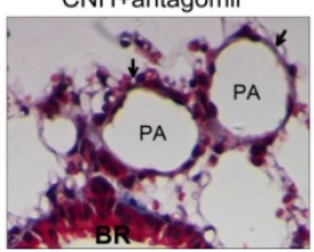

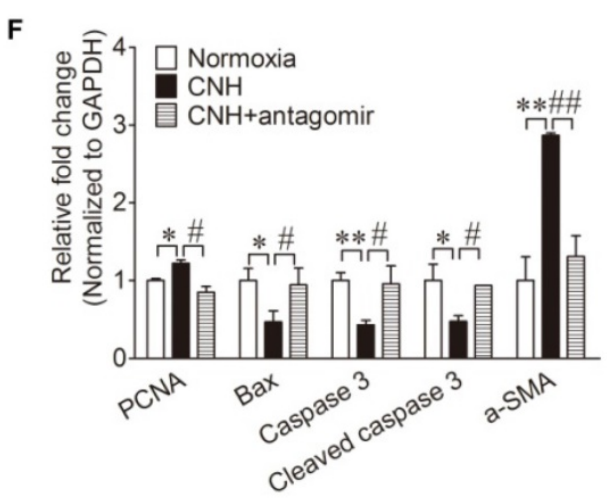

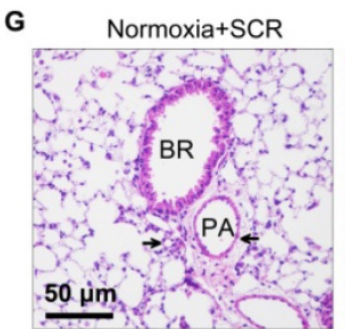

H

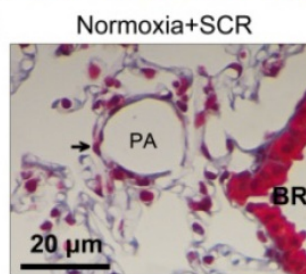

Figure 2. Blockade of miR-335-3p prevents the development of CNH-induced PAH and pulmonary vascular remodeling in mice. (A) Schematic diagram of the experimental protocol. MiR-335-3p antagomir treatment reversed CNH-induced increase of RVSP (B) and RV/LV+S (C). (D) qRT-PCR analysis of miR-335-3p expression in the lungs of mice after treatment of miR-335-3p antagomir. (E) Western blotting analysis of relative proteins expression in the lungs of mice with Normoxia, $\mathrm{CNH}$, or $\mathrm{CNH}+$ antagomir treatment. (F) Quantification of proteins expression bands in (E) was done by densitometry and normalized to GAPDH. (G) Representative images of H\&E staining of lung sections. (H) Representative masson trichrome staining of lung section. Values shown are means \pm s.e.m. $\mathrm{N}=5-8$ per group. ${ }^{*} p<0.05, * * p<0.01$ vs. Normoxia. $\# p<0.05, \# p<0.01$ vs. $\mathrm{CNH}+\mathrm{SCR}$. SCR, scramble control. PA, pulmonary artery. BR, bronchiole.

\section{Blocking miR-335-3p attenuates established hypoxic $\mathrm{PAH}$ in mice}

Data presented above (Figure 2) provide evidence that inhibition of miR-335-3p abrogates $\mathrm{CNH}$-induced $\mathrm{PAH}$ in mice. We next investigated whether blocking miR-335-3p could attenuate the progression of established hypoxic PAH in mice. To this end, mice were exposed to $\mathrm{CNH}$ for 5 weeks followed by a further 5 weeks under normoxic conditions prior to miR-335-3p antagomir (or scrambled control) administration at 11, 12, 13, and 14 weeks (Figure 3A). Results showed that $\mathrm{CNH}$ treatment for 5 weeks significantly increased RVSP from $23.3 \pm 2.1$ to $30.9 \pm 0.9 \mathrm{mmHg}$ and $\mathrm{RVH}$ index from $0.22 \pm 0.02$ to $0.26 \pm 0.01$; whereas miR-335-3p antagomir treatment significantly reduced the increased RVSP from $30.9 \pm 0.9$ to $24.9 \pm 1.1 \mathrm{mmHg}$ and $\mathrm{RVH}$ index from $0.26 \pm 0.01$ to $0.21 \pm 0.02$ (all $p<0.05 ; \mathrm{N}=5-8$ per group) (Figure 3B, Figure 3C). These findings indicate a therapeutic effect of miR-335-3p on CNH-established $\mathrm{PAH}$ in mice. 
A

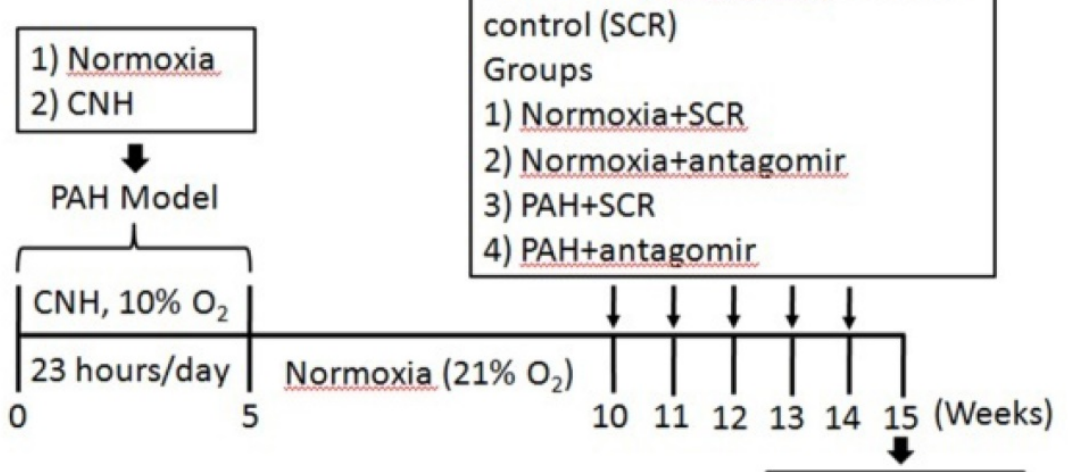

Tail vein injection miR-335-3p antagomir/scramble control (SCR)

Groups

1) Normoxia+SCR

Normoxiatantagomi

$\mathrm{PAH}+\mathrm{SCR}$

Measurements

1) RVSP

2) $R V / L V+S$
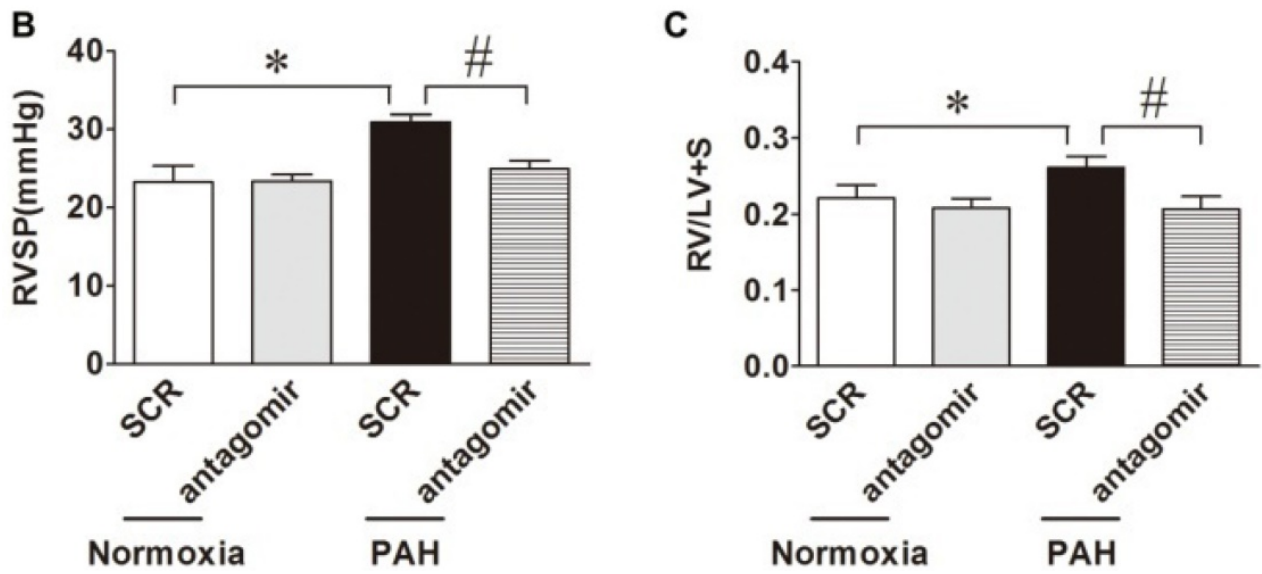

Figure 3. Blockade of miR-335-3p attenuates established hypoxic PAH in mice. (A) Schematic diagram of the experimental protocol. MiR-335-3p antagomir treatment attenuates established hypoxic PAH in mice by reversing $\mathrm{CNH}$-induced increase in RVSP (B) and RV/LV+S (C). Values shown are means \pm s.e.m. $*_{p}<0.05$ vs. Normoxia. $\# p<0.05$ vs. $C N H+S C R$. $N=5-8$ per group. SCR, scramble control.

\section{Inhibition of NF-KB activation alleviates CNH-induced PAH}

To test the role of $\mathrm{NF}-\mathrm{kB}$ in a mouse $\mathrm{CNH}$-induced PAH model, we treated mice with PDTC, an inhibitor of NF-KB, and examined the ensuing effects on $\mathrm{CNH}$-induced PAH. Two-way ANOVA indicated a significant effect of $\mathrm{CNH}$ $\left(\mathrm{F}_{(1,15)}=13.678 ; p=0.003\right)$ and $\mathrm{CNH} \times \operatorname{PDTC}\left(\mathrm{F}_{(1,15)}=5.620\right.$; $p=0.035$ ) on RVSP, but no significant effect of PDTC alone $\left(\mathrm{F}_{(1,15)}=1.729 ; p=0.213\right)$. An analogous effect of $\mathrm{CNH} \quad\left(\mathrm{F}_{(1,26)}=19.325 ; p=0.0006\right)$ and $\mathrm{CNH} \times \mathrm{PDTC}$ $\left(\mathrm{F}_{(1,26)}=4.703 ; p=0.041\right)$ but not PDTC alone $\left(\mathrm{F}_{(1,26)}=0.371 ; p=0.549\right)$ was observed also in relation to the RV/(LV+S) ratio. Post hoc analysis revealed that $\mathrm{CNH}$ exposure significantly increased RVSP and the $\mathrm{RV} /(\mathrm{LV}+\mathrm{S})$ ratio relative to normoxia control mice. Treatment of CNH-exposed mice with PDTC diminished CNH-induced increases in RVSP (Figure $4 \mathrm{~A}$ ) and the $\mathrm{RV} /(\mathrm{LV}+\mathrm{S})$ ratio (Figure $4 \mathrm{~B}$ ), indicating a preventive effect of NF-kB on $\mathrm{CNH}$-induced PAH in mice.

To further confirm whether NF-kB was involved in $\mathrm{CNH}$-induced $\mathrm{PAH}$ in mice, we assessed the effect of $\mathrm{CNH}$ on $\mathrm{PAH}$ development in $\mathrm{p} 50^{-/-}$mice. Two-way ANOVA supported a significant effect of $\mathrm{CNH} \quad\left(\mathrm{F}_{(1,15)}=5.355 ; \quad p=0.039\right), \quad \mathrm{NF}-\mathrm{kB} \quad\left(\mathrm{F}_{(1,15)}=6.670\right.$; $p=0.024)$, and $\mathrm{CNH} \times \mathrm{NF}-\mathrm{KB}\left(\mathrm{F}_{(1,15)}=4.451 ; p=0.05\right)$ on RVSP. Similarly, $\mathrm{CNH}\left(\mathrm{F}_{(1,15)}=5.031 ; p=0.04\right), \mathrm{NF}-\mathrm{kB}$ $\left(\mathrm{F}_{(1,15)}=10.369 ; \quad p=0.006\right), \quad$ and $\quad \mathrm{CNH} \times \mathrm{NF}-\mathrm{KB}$ $\left(F_{(1,15)}=14.622 ; p=0.002\right)$ exerted a significant effect on the $\mathrm{RV} /(\mathrm{LV}+\mathrm{S})$ ratio. Post hoc analysis showed that wild-type (WT) mice exposed to $\mathrm{CNH}$ for 5 weeks exhibited a significant increase in RVSP (Figure 4C) and the $\mathrm{RV} /(\mathrm{LV}+\mathrm{S}$ ) ratio (Figure $4 \mathrm{D})$ compared with mice in the normoxia control group. In contrast, the same elevated $\mathrm{CNH}$-induced parameters were significantly diminished in $\mathrm{p} 50^{-/-}$mice. These findings directly confirm that NF-kB-deficient mice are resistant to $\mathrm{CNH}$-induced $\mathrm{PAH}$. 

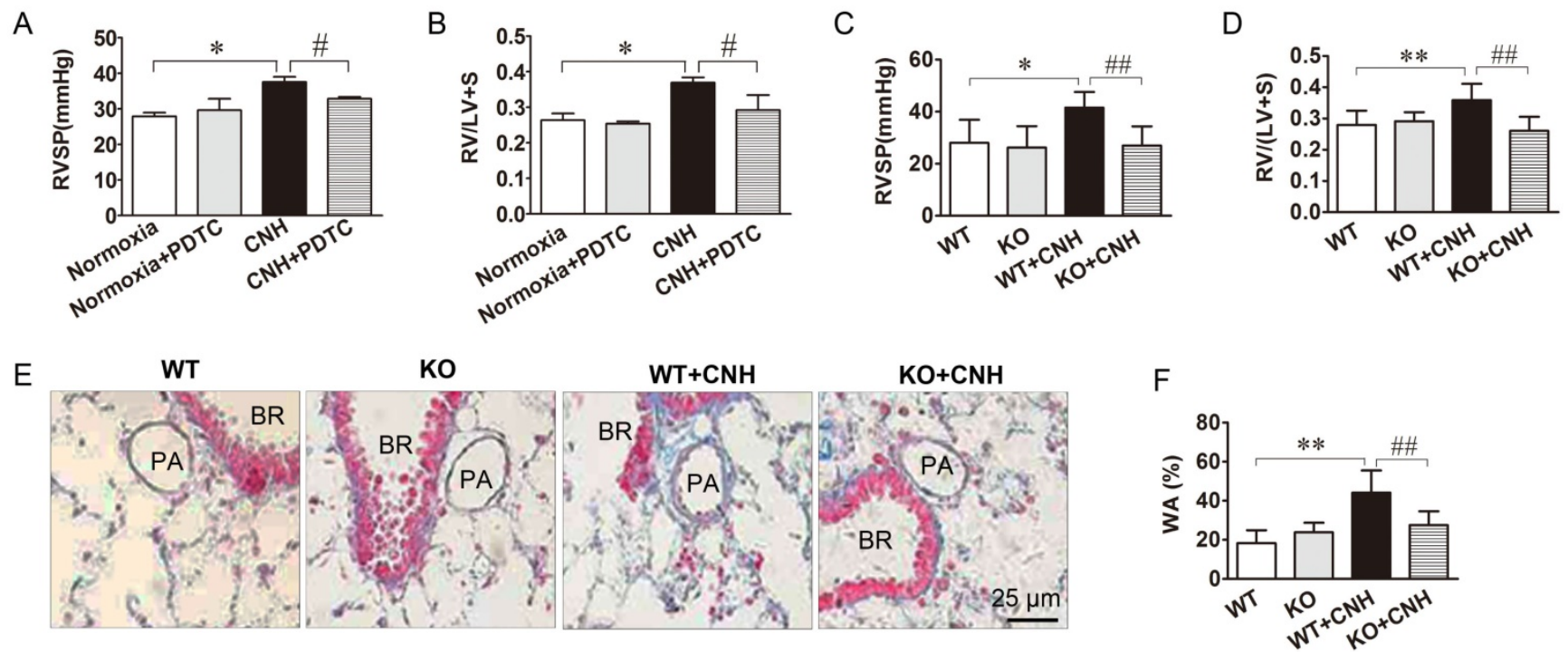

Figure 4. Blockade of NF-KB ammeliorates $\mathbf{C N H}$-induced PAH and pulmonary vascular remodeling. PDTC treatment suppressed CNH-induced increase in RVSP (A) and RV/(LV+S) (B). CNH exposure significantly increased RVSP (C) and RV/(LV+S) ratio (D) in WT but not in KO mice. (E) Representative images of the pulmonary vascular remodeling in lung sections subjected to Masson trichrome staining in WT and KO mice. (F) Quantitative analysis of pulmonary arterial wall area/total cross area (WA/TA). Values shown are means \pm s.e.m. $\mathrm{N}=5-8$ per group. $* p<0.05, * * p<0.01$ vs. Normoxia. \#p<0.05, \#\#<0.01 vs. CNH. PA, pulmonary artery. BR, bronchiole.

We next evaluated the effect of $\mathrm{CNH}$ on pulmonary artery remodeling using Masson trichrome staining in $\mathrm{p} 50^{-/-}$mice treated with $\mathrm{CNH}$ or normoxia controls. WT mice exposed to $\mathrm{CNH}$ for 5 weeks exhibited a marked increase in the wall thickness of pulmonary small distal arteries when compared with normoxic mice. This same $\mathrm{CNH}$-induced increase in wall thickness was significantly diminished in p50-/- mice (Figure 4E, Figure $4 \mathrm{~F}$ ). There was no difference between WT and p50-\% mice under normoxia. These findings clearly suggest the involvement of NF-kB in $\mathrm{CNH}$-induced pulmonary vascular remodeling in mice.

\section{MiR-335-3p is transcriptionally regulated by NF-KB}

Given that NF-kB could transcriptionally regulate downstream target miRs by binding directly to their promoters $[37,38]$, and that activated NF-kB signaling contributed to $\mathrm{CNH}$-induced $\mathrm{PAH}$ in mice, we hypothesized that $\mathrm{CNH}$-induced upregulation of miR-335-3p might be NF-KB-related. Therefore, first, we performed a database search (http://asia.ensembl.org/index.html) to identify the miR-335-3p promoter. We found that the mouse miR-335-3p gene was located on chromosome 6 and consisted of five exons separated by $\sim 17 \mathrm{~kb}$ of genomic sequence. A putative NF-KB binding site (GGGACTCTCT) was found $\sim 12 \mathrm{~kb}$ upstream of exon 1 , which was localized furthest upstream from the transcription start site (Figure 5A). To confirm the role of NF- $\mathrm{kB}$ signaling in the transcriptional regulation of miR-335-3p, we constructed a WT luciferase reporter vector containing $2260 \mathrm{bp}$ of the mouse pri-miR-335-3p 5' proximal genomic region and a mutant luciferase reporter vector, in which the NF-kB binding sites were deleted. HEK293 cells were co-transfected with the luciferase reporter vector and p65 cDNA vector. Activation of NF-kB expression increased luciferase activity driven by the WT miR-335-3p promoter, whereas p65 cDNA had no significant effect on the activity of mutant miR-335-3p promoter (Figure 5B). To further investigate the role of NF- $\mathrm{kB}$ in activating miR-335-3p transcription in the $\mathrm{CNH}$-induced $\mathrm{PAH}$ mouse model in vivo, we performed qRT-PCR analysis of pri-miR-335-3p and miR-335-3p expression in CNH mouse lungs treated with the NF-kB inhibitor PDTC. Both pri-miR-335-3p and miR-335-3p expression were significantly increased after $\mathrm{CNH}$-treatment; however, this effect was significantly suppressed by PDTC pretreatment (Figure 5C, Figure 5D). Together, these results suggest that $\mathrm{CNH}$-induced miR-335-3p upregulation depends on NF-kB binding to the primary miR-335-3p promoter.

\section{MiR-335-3p downregulates apelin-APJ signaling in mouse lungs}

MiRs mediate post-transcriptional regulation by binding directly to sequences analogous to their seed region in the 3'-UTR of target mRNAs. To identify the downstream molecular candidates of miR-335-3p, we examined the predicted miR-335-3p targets using bioinformatics tools. Using an available prediction algorithms (Targetscan), we produced a comprehensive list of all possible miR-335-3p targets. 

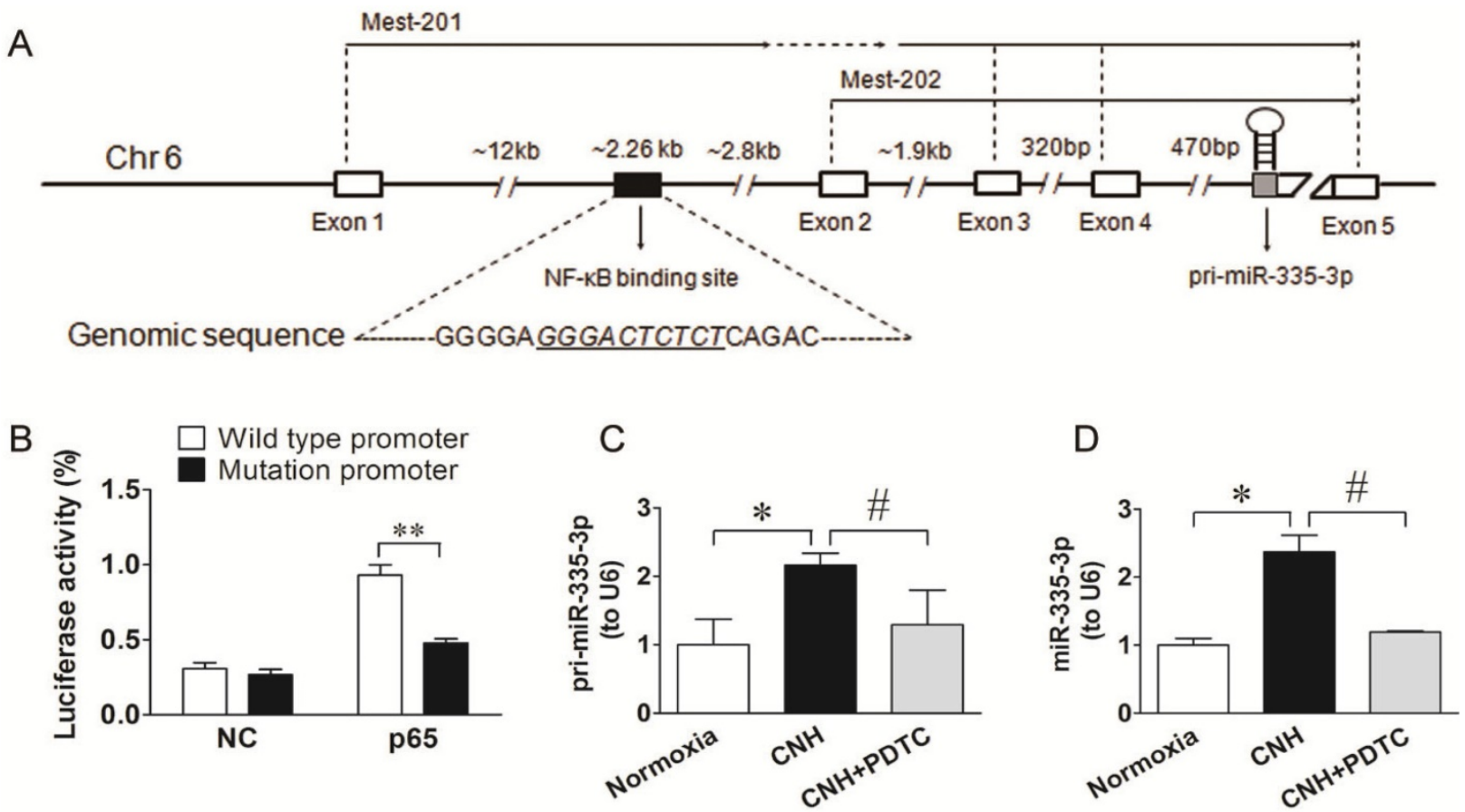

Figure 5. NF-KB is a transcriptional regulator upstream of miR-335-3p. (A) Schematic representation of putative NF-KB binding site in the promoter region of miR-335-3p, and the corresponding mutation was generated within the binding site. (B) Luciferase assay showed that the activity of the luciferase reporter fusing wild-type promoter, but not the mutated promoter with mutation within NF-KB binding site, was increased significantly after $p 65 \mathrm{cDNA}$ treatment. $* * p<0.01$. $\mathrm{NC}$ refers to an empty vector. (C) qRT-PCR analysis of pri-miR-335-3p and miR-335-3p (D) expression in the lungs of mice. Values shown are means \pm s.e.m. $\mathrm{N}=5-8$ per group. $* p<0.05$ vs. Normoxia. $\# p<0.05$ vs. $\mathrm{CNH}$.

We honed onto APJ as a potential target of miR-335-3p because it contained a conserved miR-335-3p seed sequence in its 3'-UTR (Figure 6A). Importantly, apelin and APJ have been consistently demonstrated to be critical players in PAH and were reported to localize to PASMCs [39-42]. These data led us to speculate that apelin/APJ might be functional downstream targets of miR-335-3p during $\mathrm{CNH}$-induced PAH. To verify this hypothesis, we first transfected HEK293 cells with a plasmid containing a luciferase-APJ 3'-UTR construct and found co-transfection with miR-335-3p agomir decreased luciferase activity. This finding directly confirmed the binding of miR-335-3p to the 3'-UTR of $\mathrm{APJ}$ and a consequent reduction in its transcription (Figure 6B). We then performed western blotting to test the level of APJ in CNH-treated mouse lungs. As shown in Figure 6C and Figure 6D, APJ expression was reduced in $\mathrm{CNH}$-treated mouse lungs compared with normoxia controls and, importantly, this decrease was prevented by miR-335-3p antagomir treatment. The data presented thus far provide evidence that increased levels of miR-335-3p might contribute to the pathogenesis of hypoxic PAH through repression of key cellular targets such as APJ. Taken together, our data suggest that exacerbated expression of miR-335-3p during $\mathrm{CNH}$-induced $\mathrm{PAH}$ progression, proceeds at least in part through inhibition of the apelin-APJ signaling pathway.
Apelin-13 treatment diminishes $\mathrm{CNH}$-induced $\mathrm{PAH}$ and ameliorates pulmonary artery remodeling in mouse lungs

APJ and its cognate endogenous ligand apelin are important for the development of $\mathrm{PAH}$, and disruption of the apelin-APJ axis plays a major part in the pathogenesis of PAH [39-41]. To firmly establish the mechanistic role of miR-335-3p given its impact on APJ expression, we asked whether activation of APJ in vivo could attenuate $\mathrm{CNH}$-induced $\mathrm{PAH}$. Two-way ANOVA indicated a significant effect of $\mathrm{CNH}$ $\left(\mathrm{F}_{(1,25)}=11.005 ; p=0.004\right)$ and $\mathrm{CNH} \times$ apelin $\left(\mathrm{F}_{(1,25)}=5.239\right.$; $p=0.036)$ on RVSP, while no such effect was observed for apelin alone $\left(\mathrm{F}_{(1,25)}=0.122 ; p=0.732\right)$. The same effect of $\mathrm{CNH}\left(\mathrm{F}_{(1,25)}=11.213 ; p=0.003\right)$ and $\mathrm{CNH} \times$ apelin $\left(\mathrm{F}_{(1,25)}=4.595 ; \quad p=0.043\right)$ but not apelin alone $\left(\mathrm{F}_{(1,25)}=1.275 ; p=0.27\right)$ was observed also regarding the $\mathrm{RV} /(\mathrm{LV}+\mathrm{S})$ ratio. Post hoc analysis showed that RVSP in the $\mathrm{CNH}$ group, but not in the $\mathrm{CNH} \times$ apelin-13 group, was significantly higher relative to the normoxia control group (Figure 7A). Consistent with changes in RVSP, the $\mathrm{RV} /(\mathrm{LV}+\mathrm{S})$ ratio was markedly enhanced in $\mathrm{CNH}$-exposed mice, which was strongly attenuated with apelin-13 treatment compared with $\mathrm{CNH}$-exposed mice treated with vehicle only, (Figure 7B). To determine whether $\mathrm{CNH}$ exposure could induce cellular proliferation while apelin-13 administration ameliorated this effect of $\mathrm{CNH}$, we 
assessed relative protein levels in mouse lungs. We found that PCNA and a-SMA were significantly increased while total/cleaved caspase 3 and Bax were markedly decreased in $\mathrm{CNH}$-treated mice relative to the normoxia control group; however, this effect was alleviated following apelin-13 administration (Figure 7C, Figure 7D). Concomitantly, we noted that apelin-13 treatment significantly suppressed $\mathrm{CNH}$-induced pulmonary artery remodeling (Figure 7E, Figure 7F). Together, these data indicate that amelioration of apelin-13 in $\mathrm{CNH}$-induced $\mathrm{PAH}$ in mice occurs through inhibition of cellular proliferation and pulmonary artery remodeling.

\section{Discussion}

$\mathrm{PAH}$ is characterized by progressive remodeling of the pulmonary artery, which can result in increased pulmonary vascular resistance, right heart failure, and even death. Growing evidence suggests that miRs, which act as important regulators of gene expression at the post-transcriptional level, play essential roles in $\mathrm{PAH}$ but could have also a preventive and therapeutic effect on the disease $[14,17,18]$. Our study provides the first evidence demonstrating a novel role of the $\mathrm{NF}-\mathrm{kB} / \mathrm{miR}-335-3 \mathrm{p} / \mathrm{APJ}$ axis in regulating $\mathrm{CNH}$-induced $\mathrm{PAH}$. Indeed, we report increased NF-kB activation and high miR-335-3p expression but decreased APJ expression in lung tissues of a CNH-induced PAH mouse model. Conversely, blocking NF-kB by pharmacological means resulted in a decrease in miR-335-3p but an increase in APJ expression. Similarly, repression of miR-335-3p restored APJ expression and lessened the hypoxic effect on RVSP and RVH. We further identified and confirmed NF- $\mathrm{kB}$ as a direct upstream transcriptional regulator of miR-335-3p, and APJ as a downstream target of miR-335-3p. Moreover, exogenous apelin-13 treatment diminished $\mathrm{CNH}$-induced $\mathrm{PAH}$ and ameliorated pulmonary artery remodeling. Together, our results suggest that activation of NF- $\mathrm{kB}$ leads to an upregulation of $\mathrm{miR}-335-3 \mathrm{p}$ in response to hypoxia, which promotes repression of APJ in vivo, drives pulmonary artery remodeling, and induces PAH.

A
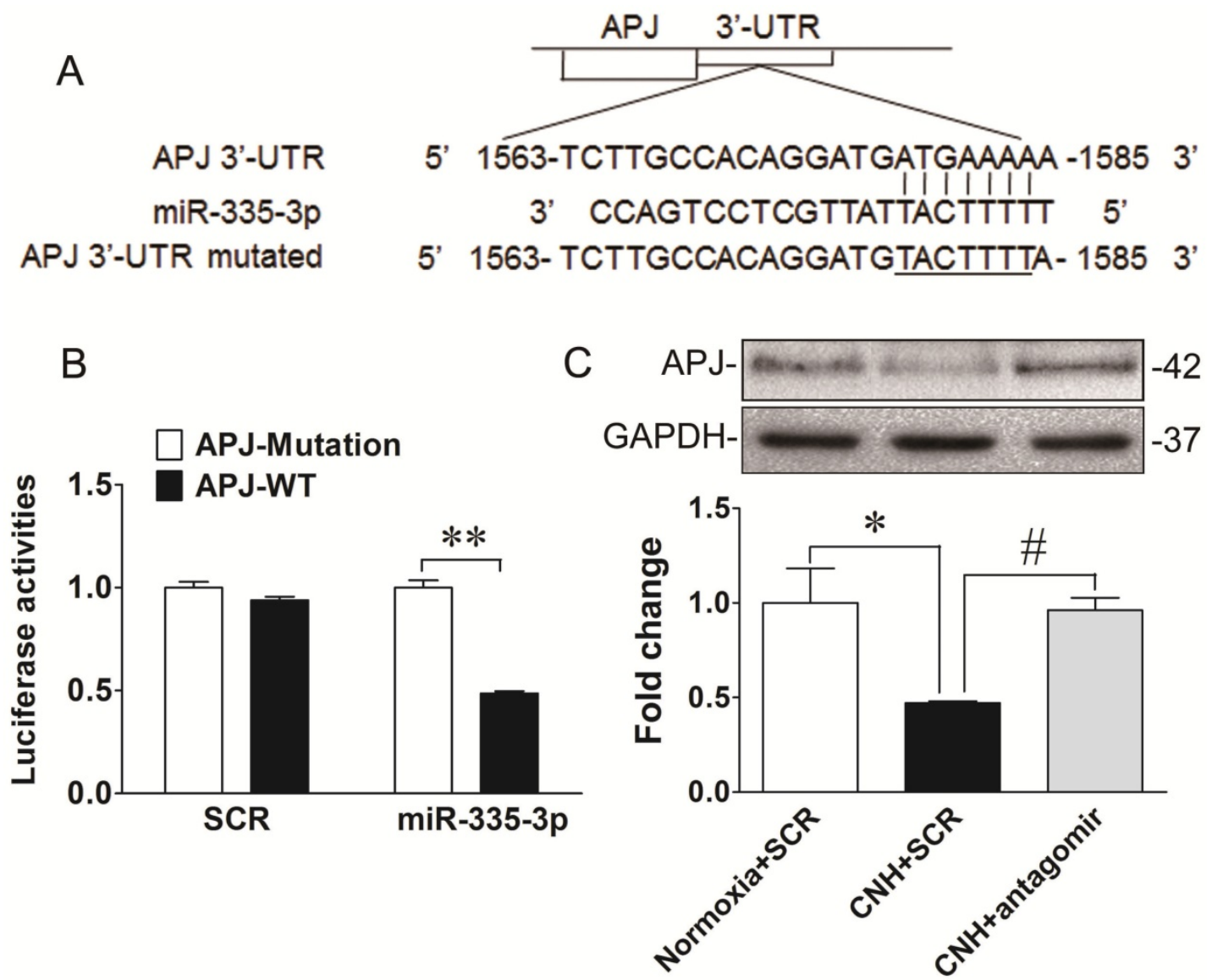

Figure 6. APJ is a direct downstream target of miR-335-3p. (A) Diagram of the miR-335-3p binding sites in the $3^{\prime}-U T R$ of the APJ gene. (B) Luciferase reporter gene assay $3^{\prime}-$ UTR of APJ with target and its mutant along with miR-335-3p agomir or scramble control vectors in HEK293 cells. **p<0.01. (C) Western blotting analysis of APJ expression in the lungs of mice. (D) Quantification of APJ expression bands in (C) was done by densitometry and normalized to GAPDH. Values shown are means \pm s.e.m. $\mathrm{N}=5-8$ per group. ${ }^{*} p<0.05$ vs. Normoxia. $\# p<0.05$ vs. $\mathrm{CNH}$. 
A

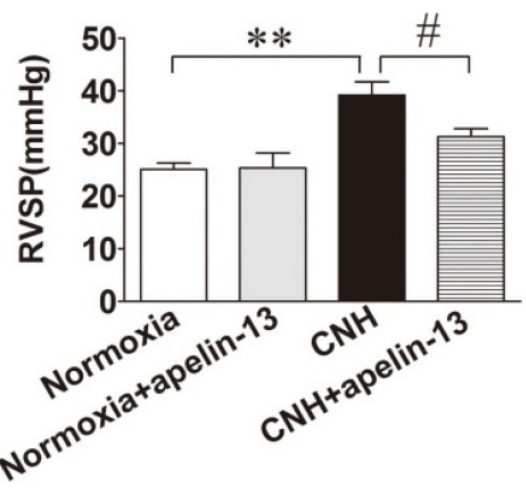

B

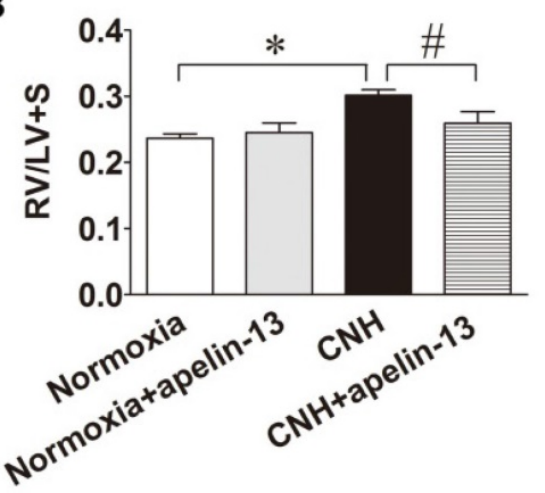

C

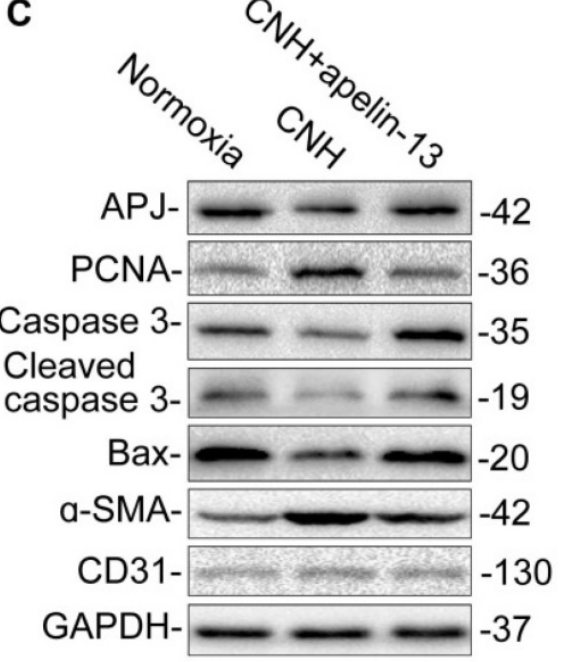

D

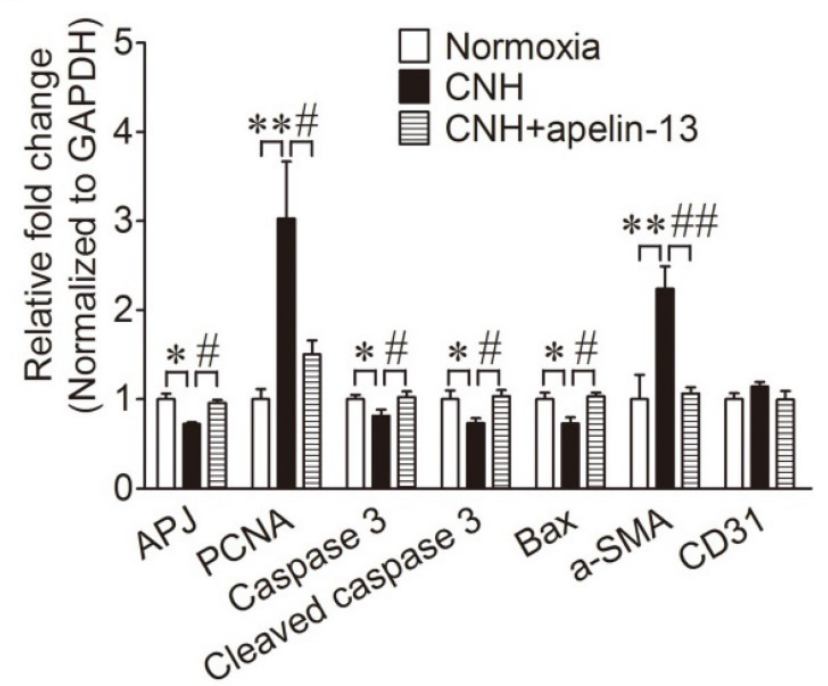

E

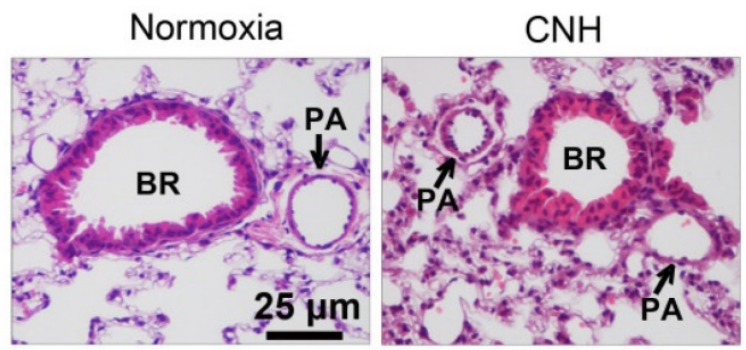

$\mathrm{CNH}+$ apelin-13

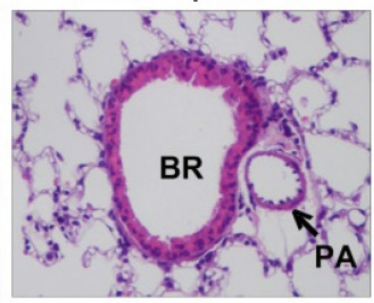

$\mathbf{F}$

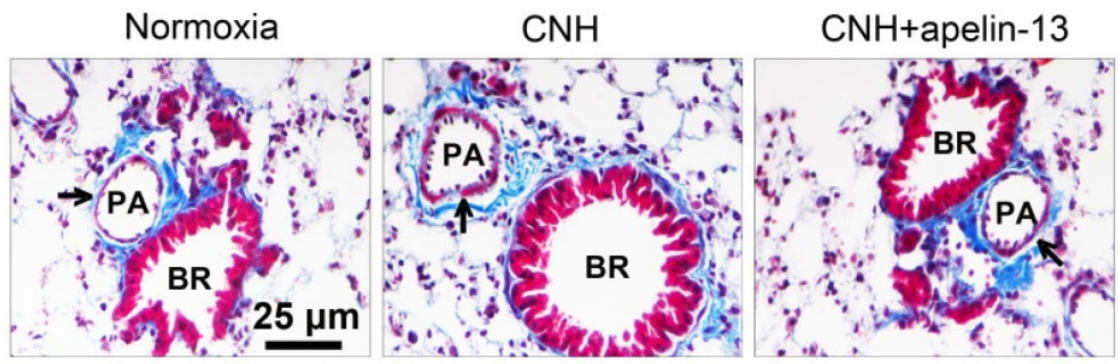

Figure 7. Amelioration of apelin-13 in CNH-induced PAH and pulmonary vascular remodeling in mice. Apelin-13 treatment suppressed $\mathrm{CNH}$-induced increase in RVSP (A) and RV/LV+S (B). (C) Western blotting analysis of relative proteins expression in the lungs of mice. (D) Quantification of proteins expression bands in (C) was done by densitometry and normalized to GAPDH. (E) Representative images of H\&E staining of lung sections. (F) Representative Masson trichrome staining of lung section. Values shown are means \pm s.e.m. $N=5-8$ per group. $* p<0.05$, $* * p<0.01$ vs. Normoxia. \#p<0.05, \# $\#<0.01$ vs. CNH. PA, pulmonary artery. BR, bronchiole. 

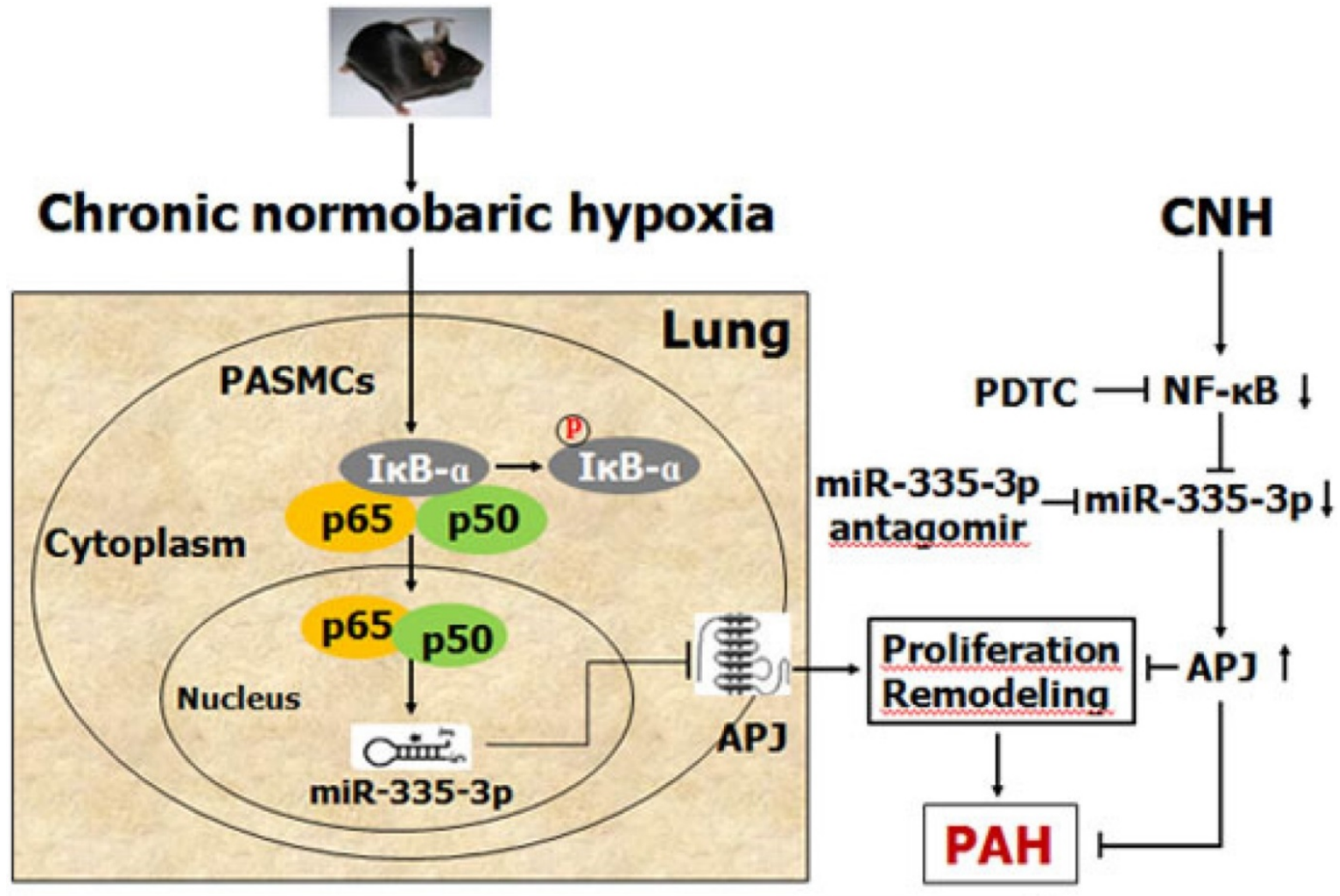

CNH

$\rightarrow$ stimulation $\rightarrow$ inhibition $\uparrow$ increase $\downarrow$ decrease

Figure 8. Schematic model showing the role and mechanism of miR-335-3p in chronic normobaric hypoxia ( $\mathrm{CNH})$-induced pulmonary arterial hypertension (PAH) in mice.

Numerous studies have indicated the involvement of miRs in regulating PAH development, sparking clinically relevant research on the regulation of endogenous miRs in PAH models [10, 13-15, 17, 18, 43]. In the present study, we first investigated the potential involvement of miRs in the regulation of $\mathrm{CNH}$-induced $\mathrm{PAH}$ by characterizing the profile of miRs expressed in mouse lung tissues following $\mathrm{CNH}$ exposure. By combining microarray and qRT-PCR results, we identified miR-335-3p to be robustly upregulated in lung tissues in a CNH-induced $\mathrm{PAH}$ mouse model. While previous studies have demonstrated that miR-335 is critically involved in the regulation of reparative activities of human mesenchymal stem cells [44] and promotes mesendodermal lineage segregation [45], none, to the best of our knowledge, has comprehensively defined the mechanistic role of miR-335-3p in PAH pathogenesis. Here, we demonstrate that blocking miR-335-3p could prevent CNH-induced PAH development and attenuate pulmonary artery remodeling. Emerging evidence suggests that the expression of miRs can be regulated at transcriptional levels [46], and NF-KB has been shown to promote miR-223-3p transcriptional induction, thus facilitating the proliferation and migration of gastric cancer cells [47]. Increased NF-KB activation was previously observed in mouse and rat lungs following hypoxia [33] or monocrotaline [27, 29] stimulation. In this study, promoter analysis led to the identification of a putative NF- $\mathrm{kB}$ binding site located in the promoter region of the miR-335-3p gene. Luciferase reporter assays clearly indicated that $\mathrm{CNH}$-induced elevation of miR-335-3p expression occurred in an NF-kB-dependent manner. In addition, by using both pharmacological and genetic approaches, we demonstrated that NF-KB was involved in $\mathrm{CNH}$-induced $\mathrm{PAH}$ because NF-KB inhibitor treatment ameliorated $\mathrm{CNH}$-induced $\mathrm{PAH}$ in mice, whereas NF-kB knockout mice were resistant to $\mathrm{CNH}$-induced $\mathrm{PAH}$. Our results are in agreement with previous reports that NF-KB activation is involved in PAH development [27-29, 33].

The post-transcriptional regulation of gene expression by miRs depends on them binding to either specific sites in the 3 '-UTR or the coding regions of their target mRNAs, resulting in degradation or translational stalling [7]. Bioinformatics miR target prediction yielded several potential targets, but the APJ gene had particularly high scores. APJ is a 
validated target of miR-335-3p and, together with its ligand apelin, is highly expressed in the pulmonary vasculature [48]. Both are also known to be potent regulators during PAH development $[39,41,42,49$, 50]. Mice with apelin deletion develop a more severe form of PAH when exposed to chronic hypoxia [40], directly confirming the involvement of the apelin-APJ axis in the pathogenesis of hypoxic PAH. We first verified and confirmed that miR-335-3p directly bound to the complementary sites on the 3 '-UTR of APJ and decreased its protein levels. Because miR-335-3p expression was markedly elevated in mouse lung tissues, we then investigated its upregulation by examining the expression of APJ. Delivery of antagomir-335-3p restored APJ expression, which was downregulated by exposure to $\mathrm{CNH}$. Finally, injection of apelin-13 attenuated $\mathrm{CNH}$-induced cellular proliferation and pulmonary arterial remodeling, and ameliorated $\mathrm{PAH}$ phenotypes (RVSP, RVH) in mice exposed to $\mathrm{CNH}$. Our data are in line with previous reports [40, 41, 49]. Notably, the apelin/APJ combination has been found to retard proliferation of PASMCs during $\mathrm{PAH}$ development [51, 52]. Pulmonary arterial endothelial cells with apelin deletion were reported to promote PASMCs proliferation; however, proliferation was suppressed and apoptosis was induced when apelin levels were restored. Moreover, apelin administration reversed $\mathrm{PAH}$ in mice with reduced production of apelin resulting from deletion of PPARY in endothelial cells [52]. In addition, apelin treatment inhibited the proliferation and migration of PASMCs mediated by the PI3K/Akt/mTOR signaling pathways under hypoxia [51]. Together, these findings suggest that miR-335-3p promotes $\mathrm{CNH}$-induced $\mathrm{PAH}$ by directly targeting APJ, while APJ exerts a protective role in the development of $\mathrm{CNH}$-induced PAH. Viable conditional $\mathrm{KO}$ mice, whose miR-335-3p gene is inactivated in the lungs, would allow further assessment of miR-335-3p function during $\mathrm{CNH}$-induced $\mathrm{PAH}$. This would advance our understanding of the exact mechanism by which miR-335-3p contributes to hypoxic PAH. Here, we propose that augmented miR-335-3p activation during $\mathrm{CNH}$ exposure plays a crucial role in promoting the development of $\mathrm{PAH}$ by targeting APJ. However, we cannot rule out the possibility that miR-335-3p exhibits its biological role by targeting other downstream genes closely associated with PAH. Additional studies are required to confirm this possibility.

In summary, this study provides the first evidence that NF-kB-mediated transcriptional upregulation of miR-335-3p expression is positively involved in the progression of $\mathrm{CNH}$-induced $\mathrm{PAH}$, and that miR-335-3p modulates the development of $\mathrm{CNH}$-induced PAH via inhibition of APJ (Figure 8). Our data highlight the critical role of the NF-kB/miR-335-3p/APJ axis in CNH-induced PAH; consequently, miR-335-3p may be a potential therapeutic target against $\mathrm{PAH}$ during hypoxia.

\section{Abbreviations}

3'-UTR: 3'-untranslated region; $\mathrm{CNH}$ : chronic normobaric hypoxia; miR: microRNA; NF-kB: nuclear factor-kappa beta; PAH: pulmonary arterial hypertension; PASMCs: pulmonary arterial smooth muscle cells; PDTC: pyrrolidine dithiocarbamate; RVH: right ventricular hypertrophy; RVSP: right ventricular systolic pressure; $\mathrm{RV} /(\mathrm{LV}+\mathrm{S})$ : right ventricle to left ventricle plus septum; WT: wild-type.

\section{Supplementary Material}

Supplementary figure S1.

http://www.ijbs.com/v16p0515s1.pdf

\section{Acknowledgements}

This work was supported by the Zhejiang Provincial Natural Science Foundation of China (LY20H010001, J.M. Fan; LY17H010007, X.F. Fan; LY18H010007, Y.S. Gong; LY14H010005, S.Z. Mao) and the National Natural Science Foundation of China (81200039, X.F. Fan). We sincerely thank Professor Shufang Liu (Institute of Hypoxia Medicine, Wenzhou Medical University) for his helpful editing the manuscript and discussion.

\section{Ethics Approval}

All experimental procedures were performed in accordance with the guidelines for animal care and were approved by the Animal Ethics Committee of Wenzhou Medical University, and complied with the National Institutes of Health guide for the Laboratory animals (NIH Publications No. 80-23, revised 1996).

\section{Author Contributions}

JM Fan, YS Gong, and XF Fan contributed to experiment design. JM Fan and H Guang contributed to data acquisition and analysis as well as manuscript writing. ZH Gan and YY Wang contributed to editing the manuscript. H Quan, XQ Shan, R Chen, QY Tian, FK Zhang, FZ Ye, HZ Zhang, L Ding, F Xue, SZ Mao, and LG $\mathrm{Hu}$ contributed to conducting the experimental research. All authors contributed to and have approved the final manuscript.

\section{Competing Interests}

The authors have declared that no competing interest exists. 


\section{References}

1. Badesch DB, Champion HC, Sanchez MA, et al. Diagnosis and assessment of pulmonary arterial hypertension. J Am Coll Cardiol. 2009; 54: S55-66.

2. Lai YC, Potoka KC, Champion HC, et al. Pulmonary arterial hypertension: the clinical syndrome. Circ Res. 2014; 115: 115-130.

3. Morrell NW, Adnot S, Archer SL, et al. Cellular and molecular basis of pulmonary arterial hypertension. J Am Coll Cardiol. 2009; 54: S20-31.

4. Liang OD, So EY, Egan PC, et al. Endothelial to haematopoietic transition contributes to pulmonary arterial hypertension. Cardiovasc Res. 2017; 113: 1560-1573.

5. Stenmark KR, Frid MG, Graham BB, et al. Dynamic and diverse changes in the functional properties of vascular smooth muscle cells in pulmonary hypertension. Cardiovasc Res. 2018; 114: 551-564.

6. Lagos-Quintana M, Rauhut R, Lendeckel W, et al. Identification of novel genes coding for small expressed RNAs. Science. 2001; 294: 853-858.

7. Bartel DP. MicroRNAs: genomics, biogenesis, mechanism, and function. Cell. 2004; 116: 281-97.

8. Feng SP, Cong SJ, Zhang X, et al. MicroRNA-192 targeting retinoblastoma 1 inhibits cell proliferation and induces cell apoptosis in lung cancer cells. Nucleic Acids Res. 2011; 39: 6669-6678.

9. Wang DR, Zhang H, Li M, et al. MicroRNA-124 controls the proliferative, migratory, and inflammatory phenotype of pulmonary vascular fibroblasts. Circ Res. 2014; 114: 67-78.

10. Zhou GF, Chen TJ, Raj JU. MicroRNAs in pulmonary arterial hypertension. Am J Respir Cell Mol Biol. 2015; 52: 139-151.

11. Bienertova-Vasku J, Novak J, Vasku A. MicroRNAs in pulmonary arterial hypertension: pathogenesis, diagnosis and treatment. J Am Soc Hypertens. 2015; 9: 221-234

12. Fan JM, Fan XF, Li Y, et al. Chronic Normobaric Hypoxia Induces Pulmonary Hypertension in Rats: Role of NF-kappaB. High Alt Med Biol. 2016; 17: 43-49.

13. Luo Y, Dong HY, Zhang B, et al. miR-29a-3p attenuates hypoxic pulmonary hypertension by inhibiting pulmonary adventitial fibroblast activation. Hypertension. 2015; 65: 414-420.

14. Rothman AM, Arnold ND, Pickworth JA, et al. MicroRNA-140-5p and SMURF1 regulate pulmonary arterial hypertension. J Clin Invest. 2016; 126: 2495-2508.

15. Sarkar J, Gou DM, Turaka P, et al. MicroRNA-21 plays a role in hypoxia-mediated pulmonary artery smooth muscle cell proliferation and migration. Am J Physiol Lung Cell Mol Physiol. 2010; 299: L861-871.

16. Stenmark KR, Fagan KA, Frid MG. Hypoxia-induced pulmonary vascular remodeling: cellular and molecular mechanisms. Circ Res. 2006; 99: 675-691.

17. Yang SZ, Banerjee S, Freitas A, et al. miR-21 regulates chronic hypoxia-induced pulmonary vascular remodeling. Am J Physiol Lung Cell Mol Physiol. 2012; 302: L521-529.

18. Courboulin A, Paulin R, Giguere NJ, et al. Role for miR-204 in human pulmonary arterial hypertension. J Exp Med. 2011; 208: 535-548.

19. Zeng Y, Zhang XY, Kang K, et al. MicroRNA-223 attenuates hypoxia-induced vascular remodeling by targeting RhoB/MLC2 in pulmonary arterial smooth muscle cells. Sci Rep. 2016; 6: 24900 .

20. Deng L, Blanco FJ, Stevens H, et al. MicroRNA-143 Activation regulates smooth muscle and endothelial cell crosstalk in pulmonary arterial hypertension. Circ Res. 2015; 117: 870-883.

21. Shan FB, Li JX, Huang OY. HIF-1 alpha-induced up-regulation of miR-9 contributes to phenotypic modulation in pulmonary artery smooth muscle cells during hypoxia. J Cell Physiol. 2014; 229: 1511-1520.

22. Zeng Y, Liu HT, Kang K, et al. Hypoxia inducible factor-1 mediates expression of miR-322: potential role in proliferation and migration of pulmonary arterial smooth muscle cells. Sci Rep. 2015; 5: 12098.

23. Bazzoni $F$, Rossato $M$, Fabbri $M$, et al. Induction and regulatory function of miR-9 in human monocytes and neutrophils exposed to proinflammatory signals. Proc Natl Acad Sci U S A. 2009; 106: 5282-5287.

24. Li X, Gao L, Cui Q, et al. Sulindac inhibits tumor cell invasion by suppressing NF-kappaB-mediated transcription of microRNAs. Oncogene. 2012; 31: 4979-4986.

25. Xu Z, Xiao SB, Xu P, et al. miR-365, a novel negative regulator of interleukin-6 gene expression, is cooperatively regulated by $\mathrm{Sp} 1$ and NF-kappaB. J Biol Chem. 2011; 286: 21401-21412.

26. Boldin MP, Baltimore D. MicroRNAs, new effectors and regulators of NF-kappaB. Immunol Rev. 2012; 246: 205-220.

27. Sawada H, Mitani $Y$, Maruyama J, et al. A nuclear factor-kappaB inhibitor pyrrolidine dithiocarbamate ameliorates pulmonary hypertension in rats. Chest. 2007; 132: 1265-1274

28. Huang J, Kaminski PM, Edwards JG, et al. Pyrrolidine dithiocarbamate restores endothelial cell membrane integrity and attenuates monocrotaline-induced pulmonary artery hypertension. Am J Physiol Lung Cell Mol Physiol. 2008; 294: L1250-1259.

29. Li L, Wei CY, Kim IK, et al. Inhibition of nuclear factor-kappaB in the lungs prevents monocrotaline-induced pulmonary hypertension in mice. Hypertension. 2014; 63: 1260-1269.

30. Hosokawa S, Haraguchi G, Sasaki A, et al. Pathophysiological roles of nuclear factor kappaB (NF-kB) in pulmonary arterial hypertension: effects of synthetic selective NF-kB inhibitor IMD-0354. Cardiovasc Res. 2013; 99: 35-43.
31. Price LC, Caramori G, Perros F, et al. Nuclear factor kappa-B is activated in the pulmonary vessels of patients with end-stage idiopathic pulmonary arterial hypertension. PLoS One 2013; 8: e75415.

32. Kimura S, Egashira K, Chen L, et al. Nanoparticle-mediated delivery of nuclear factor kappaB decoy into lungs ameliorates monocrotaline-induced pulmonary arterial hypertension. Hypertension. 2009; 53: 877-883.

33. Farkas D, Alhussaini AA, Kraskauskas D, et al. Nuclear factor kappaB inhibition reduces lung vascular lumen obliteration in severe pulmonary hypertension in rats. Am J Respir Cell Mol Biol. 2014; 51: 413-425.

34. Fan JM, Guang H, Zhang HZ, et al. SIRT1 mediates apelin-13 in ameliorating chronic normobaric hypoxia-induced anxiety-like behavior by suppressing NF-kappaB pathway in mice hippocampus. Neuroscience. 2018; 381: 22-34.

35. Mao SZ, Fan XF, Xue F, et al. Intermedin modulates hypoxic pulmonary vascular remodeling by inhibiting pulmonary artery smooth muscle cell proliferation. Pulm Pharmacol Ther. 2014; 27: 1-9.

36. Langmead B, Salzberg SL. Fast gapped-read alignment with Bowtie 2. Nat Methods. 2012; 9: 357-359.

37. Ambros $\mathrm{V}$. The functions of animal microRNAs. Nature. 2004; 431: 350-355

38. Kumar V, Palermo R, Talora C, et al. Notch and NF-kB signaling pathways regulate miR-223/FBXW7 axis in T-cell acute lymphoblastic leukemia. Leukemia. 2014; 28: 2324-2335.

39. Andersen CU, Hilberg O, Mellemkjaer S, et al. Apelin and pulmonary hypertension. Pulm Circ. 2011; 1: 334-346.

40. Chandra SM, Razavi H, Kim J, et al. Disruption of the apelin-APJ system worsens hypoxia-induced pulmonary hypertension. Arterioscler Thromb Vasc Biol. 2011; 31: 814-820.

41. Kim J. Apelin-APJ signaling: a potential therapeutic target for pulmonary arterial hypertension. Mol Cells. 2014; 37: 196-201.

42. Kim J, Kang YJ, Kojima Y, et al. An endothelial apelin-FGF link mediated by miR-424 and miR-503 is disrupted in pulmonary arterial hypertension. Nat Med. 2013; 19: 74-82.

43. Meloche J, Le Guen M, Potus F, et al. miR-223 reverses experimental pulmonary arterial hypertension. Am J Physiol Cell Physiol. 2015; 309: C363-372

44. Tome M, Sepulveda JC, Delgado M, et al. miR-335 correlates with senescence/aging in human mesenchymal stem cells and inhibits their therapeutic actions through inhibition of AP-1 activity. Stem Cells. 2014; 32: 2229-2244.

45. Yang DP, Lutter D, Burtscher I, et al. miR-335 promotes mesendodermal lineage segregation and shapes a transcription factor gradient in the endoderm. Development. 2014; 141: 514-525.

46. Zhou R, Hu GK, Gong AY, et al. Binding of NF-kappaB p65 subunit to the promoter elements is involved in LPS-induced transactivation of miRNA genes in human biliary epithelial cells. Nucleic Acids Res. 2010; 38: 3222-3232.

47. Yang $\mathrm{FH}, \mathrm{Xu} \mathrm{YG}$, Liu C, et al. NF-kappaB/miR-223-3p/ARID1A axis is involved in Helicobacter pylori CagA-induced gastric carcinogenesis and progression. Cell Death Dis. 2018; 9: 12.

48. Hosoya M, Kawamata Y, Fukusumi S, et al. Molecular and functional characteristics of APJ. Tissue distribution of mRNA and interaction with the endogenous ligand apelin. J Biol Chem. 2000; 275: 21061-21067.

49. Falcao-Pires I, Goncalves N, Henriques-Coelho T, et al. Apelin decreases myocardial injury and improves right ventricular function in monocrotaline-induced pulmonary hypertension. Am J Physiol Heart Circ Physiol. 2009; 296: H2007-2014.

50. Yang PR, Read C, Kuc RE, et al. Elabela/Toddler Is an Endogenous Agonist of the apelin APJ receptor in the adult cardiovascular system, and exogenous administration of the peptide compensates for the downregulation of its expression in pulmonary arterial hypertension. Circulation 2017; 135: $1160-1173$

51. Zhang HY, Gong YS, Wang ZG, et al. Apelin inhibits the proliferation and migration of rat PASMCs via the activation of PI3K/Akt/mTOR signal and the inhibition of autophagy under hypoxia. J Cell Mol Med. 2014; 18: 542-553.

52. Alastalo TP, Li ML, Perez Vde J, et al. Disruption of PPARgamma/beta-catenin-mediated regulation of apelin impairs BMP-induced mouse and human pulmonary arterial EC survival. J Clin Invest. 2011; 121: 3735-3746. 